\section{Descrição metodológica do uso de acelerometria para mensurar a prática de atividade física nas coortes de nascimentos de Pelotas, Rio Grande do Sul, Brasil, 1993 e 2004}

\author{
Methodological description of accelerometry for \\ measuring physical activity in the 1993 and 2004 \\ Pelotas (Brazil) birth cohorts
}

\author{
Descripción metodológica del uso de \\ acelerómetros para medir la actividad física en las \\ cohortes de nacimiento de Pelotas, Rio Grande do \\ Sul, Brasil, 1993 y 2004
}

\begin{abstract}
The aim of this study was to characterize the methodology of data collection on physical activity using accelerometry in two birth cohorts (2004 and 1993) in Pelotas, Rio Grande do Sul State, Brazil, at the 6-7 and 18-year follow-up visits, respectively. During visits to the study headquarters for a health evaluation, cohort subjects received the accelerometer to be worn on the wrist for 5 to 8 days, after which the device was retrieved at their homes. Genea and GENEActiv triaxial estimators of gravity (g) acceleration were employed. Accelerometry data were collected from 3,331 children (93.7\% of those included in follow-up) and 3,816 adolescents (99\% of those in followup). The study characterizes the data collection methodology in more than 7,000 individuals and discusses issues in its implementation. It thus provides a methodological framework aimed at helping to plan future population-based studies with the use of such technology and to improve understanding of physical activity in the context of epidemiological studies.
\end{abstract}

Cohort Studies; Motor Activity; Methodology
Alan Goularte Knuth 1

Maria Cecília F. Assunção 2

Helen Gonçalves ${ }^{2}$

Ana Maria B. Menezes 2

Iná S. Santos 2

Aluísio J. D. Barros 2

Alicia Matijasevich 2

Virgílio Viana Ramires 2

Inácio Crochemore Mohnsam da Silva ${ }^{2}$

Pedro Curi Hallal 2

\section{Resumo}

O objetivo deste trabalho é caracterizar a metodologia de coleta de atividade física por meio de acelerometria nas coortes de nascidos em 2004 e 1993 em Pelotas, Rio Grande do Sul, Brasil, nos acompanhamentos dos 6-7 e 18 anos, respectivamente. Ao visitarem a sede para um amplo estudo de saúde, esses indivíduos receberam o acelerômetro e a posterior busca foi realizada no domicílio por meio de motociclistas da equipe de pesquisa. Os modelos utilizados foram o GENEA e GENEActiv, estimadores triaxiais da aceleração da gravidade (g), utilizados no punho de crianças e adolescentes por um período de 5 a 8 dias. O número de indivíduos com dados de acelerometria nas coortes foi de 3.331 crianças (93,7\% do acompanhamento) e 3.816 jovens (92,9\% do acompanhamento). Ao caracterizarmos a coleta de acelerometria em mais de 7.000 indivíduos, apresenta-se um arcabouço metodológico para o planejamento de novos estudos populacionais no tema, descrevendo situações específicas dessa experiência e qualificando a compreensão da atividade física no contexto de estudos epidemiológicos.

Estudos de Coortes; Atividade Motora; Metodologia 


\section{Introdução}

O interesse em medir com a maior acurácia possível a prática de atividade física de forma que possibilite verificar sua relação com eventos do processo saúde/doença coloca novos procedimentos referentes às medidas no centro da discussão científica na área de epidemiologia. Conforme Sirard \& Pate 1, as medidas de atividade física são classificadas em primárias ou padrão, secundárias ou objetivas e subjetivas. As medidas primárias como calorimetria indireta ou água duplamente marcada são infrequentes em estudos populacionais, por conta do custo e complexidade logística. As medidas objetivas mensuram a atividade física por meio de aparelhos voltados ao registro de movimentos corporais, como os pedômetros (avaliando o número de passos) e os acelerômetros (avaliando a aceleração produzida pelo corpo humano). As principais diferenças entre essas duas medidas são o tipo de atividade captada e a informação fornecida. Enquanto os pedômetros se restringem a mensurar a quantidade de passos, impossibilitando estimativas de intensidades ou mesmo de períodos de realização das atividades, os acelerômetros captam uma maior diversidade de movimentos, podendo distinguir também período de utilização, frequência, duração e até mesmo intensidade da atividade física. Por fim, as medidas subjetivas historicamente têm recebido o maior enfoque nos estudos epidemiológicos e são representadas especialmente pelos questionários. Depois de algum período de pesquisas baseadas majoritariamente em mensuração de atividade física por intermédio de questionários, a acelerometria surge como um avanço na forma de medida objetiva da atividade física, configurando-se na principal alternativa metodológica.

Nesse sentido, decisões metodológicas e logísticas passam a ter um papel fundamental na qualidade de aquisição dos dados e desenho de novos estudos. Aspectos como o tempo total de mensuração, o tipo de acelerômetro utilizado e suas particularidades, dias de semana ou fim de semana a serem utilizados na coleta precisam ser considerados, tanto por decisões científicas quanto pelas adequações logísticas e financeiras. Na área da acelerometria, ainda que haja um elevado número de publicações que tratam das características dos acelerômetros e seus respectivos estudos de validação em pequenas amostras 2 , há poucos estudos que ofereçam um detalhamento dos aspectos logísticos em pesquisas com abordagem populacional. Contudo, é importante avaliar também a adequação dos aparelhos às pesquisas com grandes amostras. Existem certos modelos de acelerômetros que têm aplicabilidade duradoura e se adéquam a coletas longas e com grande contingente populacional; há modelos, porém, que não suportam tal carga de trabalho.

Além do alto custo financeiro da acelerometria, se comparado ao emprego dos questionários validados, há muitos aspectos que tornam complexa a utilização de medidas objetivas de atividade física. $\mathrm{O}$ estudo de Reichert et al. ${ }^{3}$ apresentou diversas situações metodológicas envolvendo a coleta de dados de atividade física e composição corporal em uma amostra da coorte dos nascidos em 1993 de Pelotas, Rio Grande do Sul, Brasil. Nesse estudo, a coleta por acelerometria realizada em 498 jovens, com o modelo Actigra$p h$, ainda é considerado de pequena escala, em comparação, por exemplo, a estudos de coortes de nascimentos, que monitoram a saúde de um contingente bem maior de pessoas.

Em Pelotas, existem três estudos de coortes de nascimentos, contemplando os nascidos nos anos de 1982, 1993 e 2004. Publicações anteriores descrevem a metodologia em detalhes e resultados dessas coortes 4,5,6,7,8. No campo da atividade física, foi possível caracterizar o tema de forma mais enfática ao longo dos acompanhamentos. Foram utilizadas desde questões genéricas sobre visita de crianças a praças e parques nos primeiros acompanhamentos da coorte de 1982, até instrumentos padronizados, como o Questionário Internacional de Atividade Física (IPAQ) em anos posteriores e de uma versão adaptada ${ }^{9}$ desse instrumento na coorte de 1993. Maiores detalhes sobre as hipóteses testadas em tais estudos, os principais achados e o impacto nessa área podem ser encontrados nos trabalhos de Domingues et al. 10, Dumith et al. 11 e Hallal et al. 12. A partir de 2010, a utilização da acelerometria como medida objetiva de atividade física em todos os membros da coorte foi um dos avanços preconizados para os estudos dessas três populações específicas.

Assim, o acompanhamento dos 6-7 anos da coorte dos nascidos em 2004 foi o pioneiro ao experimentar a utilização da acelerometria com grandes amostras, seguido da coorte de 1993, que fez uso do instrumento no acompanhamento dos adolescentes aos 18 anos de idade, em 2010-11. Nesse sentido, o objetivo do presente artigo é caracterizar as coletas de acelerometria conduzidas em duas coortes populacionais, apresentando e discutindo os aspectos metodológicos, as dificuldades e os avanços logísticos desta abordagem. 


\section{Metodologia}

\section{Coortes de nascimento de Pelotas}

As coortes de nascimentos de Pelotas são constituídas pelos nascidos vivos de mães residentes na área urbana do município nos anos de 1982, 1993 e 2004.

Em termos de atividade física, foram utilizados vários instrumentos de coleta de dados nas três coortes populacionais contemplando o IPAQ, questionários propostos pelos pesquisadores e instrumentos baseados na percepção das mães sobre o comportamento das crianças. $\mathrm{Na}$ coorte de nascimentos de 1982 foram utilizados questionários específicos sobre atividade física aos 15 ( $\mathrm{n}=1.076), 18(\mathrm{n}=2.250)$ e 23 anos ( $\mathrm{n}=4.296$ ), mas, no último, toda a coorte foi avaliada com a utilização da versão longa do IPAQ. Na coorte de nascimentos de 1993, coletas integrais por meio de questionário foram realizadas aos $11(n=4.452)$ e aos 15 anos $(n=4.325)$. Já as coletas em pequenas amostras foram realizadas aos $4(n=634)$ e 13 anos $(n=511)$. No acompanhamento dos quatro anos, a coleta de atividade física foi realizada com a utilização de um questionário que tem a percepção da mãe sobre o comportamento da criança como o principal componente de avaliação. O mesmo questionário foi utilizado na coorte de nascimentos de 2004, também aos 4 anos ( $\mathrm{n}=3.789$ ).

Até 2010, esses estudos foram realizados quase sempre nos domicílios dos indivíduos. Em função da constituição de uma sede para fins de pesquisa, a forma de coleta das informações foi alterada e os acompanhamentos das coortes passaram a ser realizados na sede do Centro de Pesquisas Epidemiológicas da Universidade Federal de Pelotas (e, apenas complementarmente, em visitas domiciliares). Pelo acompanhamento dos 6-7 anos da coorte de 2004, realizado entre os anos de 2010 e 2011, os membros das coortes de nascimento passaram a ser convidados a comparecer nas novas instalações para os estudos e avaliações de saúde. Nesse contexto se insere a mensuração de atividade física mediante uso de acelerômetro, objeto de estudo do presente artigo.

\section{Acelerômetros utilizados}

O início da coleta na coorte de 2004, no ano de 2010, foi conduzido com o acelerômetro modelo GENEA (Gravity Estimator of Normal Everyday Activity), monitor desenvolvido e fabricado por empresa do Reino Unido. Esse modelo é um estimador da aceleração da gravidade, fornecendo resultados em "g" ( $\left.1 \mathrm{~g}=9.81 \mathrm{~m} . \mathrm{s}^{-2}\right)$ e com capa- cidade de registro programado para medição de $85 \mathrm{~Hz}$. Os arquivos gerados podem ser armazenados nos formatos BIN ou CSV, e o monitor pode ser utilizado no punho, quadril e tornozelo. Durante os acompanhamentos das coortes de 2004 e 1993, as coletas ocorreram com o monitor posicionado no punho não dominante. $\mathrm{O}$ acelerômetro é triaxial e tem a propriedade de ser à prova d'água. O carregamento de energia do acelerômetro e o download das informações captadas são realizados por meio de cabo USB simples. O modelo GENEActiv, com características bastante similares ao mencionado anteriormente, também foi utilizado durante o acompanhamento da coorte de 2004 e ao longo de toda a coleta da coorte de 1993. O GENEActiv foi projetado para superar as limitações práticas e técnicas impostas pelo modelo anterior (a serem descritas na seção de resultados).

Os acelerômetros utilizados nas coletas foram adquiridos por meio de convênio entre a Universidade Federal de Pelotas e o Medical Research Council (Epidemiology Unit) da Universidade de Cambridge, Reino Unido. O valor unitário dos monitores custa cerca de R\$ 450,00.

\section{Acompanhamento das coortes de 2004 e 1993}

Antes do início de cada coleta, uma rotina de treinamentos e testes foi realizada com intuito de prever materiais, adequar espaços físicos para o trabalho de campo, conhecimento dos monitores e seu funcionamento. A coleta de acelerometria foi mais um componente de avaliação da saúde dos indivíduos das coortes.

Os acompanhamentos dos 6-7 anos de idade da coorte de 2004 e o dos 18 anos da coorte de 1993 foram realizados na sede dos estudos. Durante a visita à sede e após leitura do Termo de Consentimento Livre e Esclarecido, os acompanhados eram encaminhados aos diversos procedimentos de pesquisa, que incluíram testes e exames, como medidas de composição corporal, ultrassom de carótidas, coleta de sangue e/ ou saliva, espirometria, pressão arterial, testes psicológicos e questionários sobre alimentação e saúde. Após a realização desses procedimentos, iniciava-se o processo de coleta de dados de acelerometria (Figura 1). Os acompanhamentos foram aprovados pelo Comitê de Ética em Pesquisa da Faculdade de Medicina da Universidade Federal de Pelotas, sob os registros 05/11 para a coorte de 1993 e 35/10 para a coorte de 2004.

As crianças (acompanhadas por seus responsáveis) e os jovens recebiam o acelerômetro e uma série de orientações sobre o uso do aparelho e os cuidados com ele. Tais instruções, na coorte 


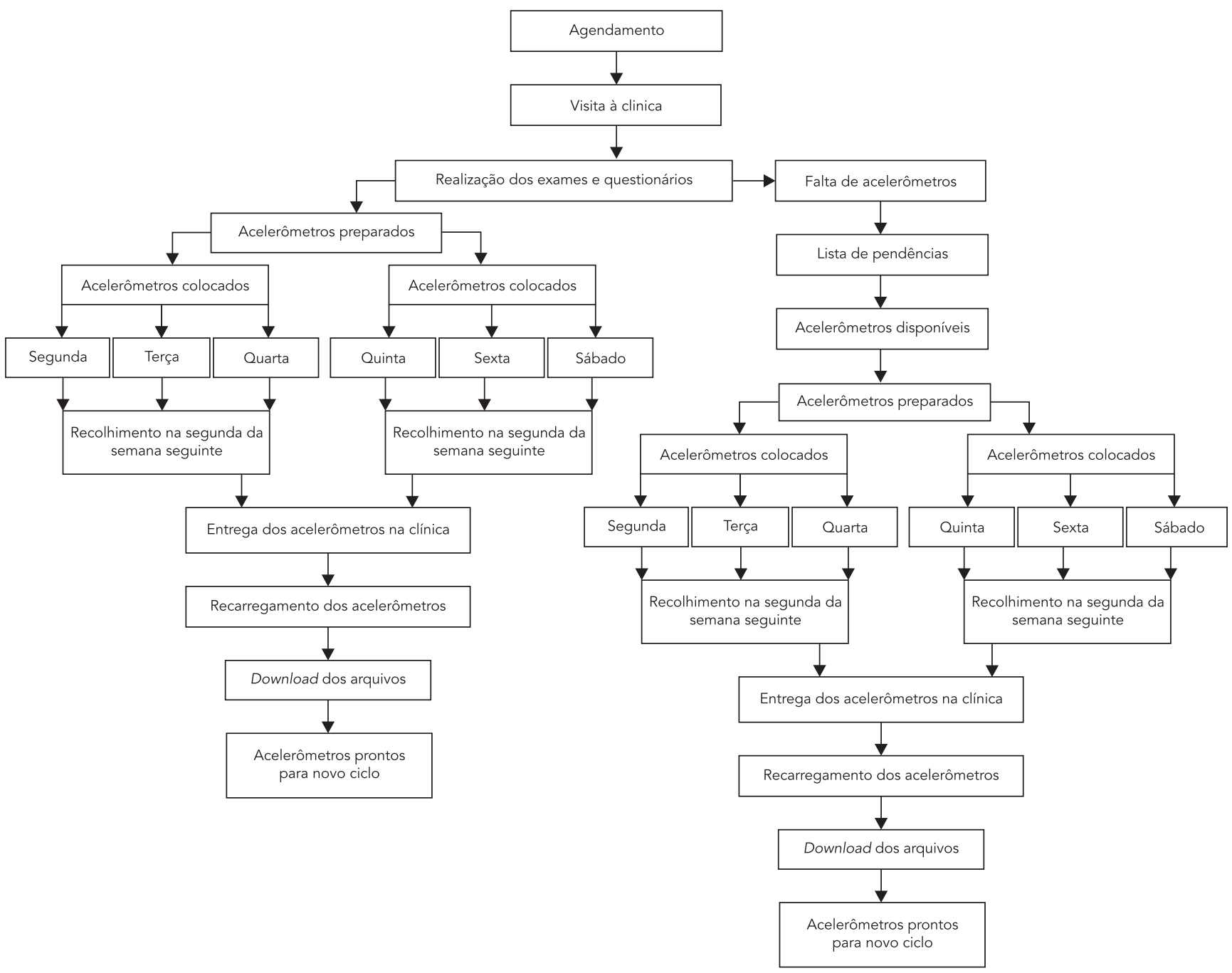

de 2004, eram fornecidas às mães e às próprias crianças. Já no acompanhamento da coorte de 1993, as informações eram passadas diretamente aos jovens. As principais recomendações incluíam: utilizar o monitor todo o período de coleta (24 horas/dia), mesmo durante o banho e nas horas de sono; ter cuidado por tratar-se de um aparelho frágil; reportar-se à equipe de estudo em caso de dúvidas ou incômodos com o uso; liberdade para readequar a pulseira do monitor; flexibilidade para interromper o uso em função de a criança/jovem se negar a completar a coleta. Por fim, um documento reforçando essas con- siderações e com telefones de contato e agendamento da busca do monitor foram entregues aos jovens e a todos os responsáveis das crianças. Por se tratar de um aparelho frágil e leve (16g), todos foram orientados a reportar-se à equipe de estudo sempre que alguma situação pudesse comprometer a coleta de informações, independentemente do horário. Logo, os pesquisadores responsáveis pela acelerometria mantinham disponibilidade permanente, por telefone, fora dos horários de funcionamento da sede.

A coleta dos monitores de atividade física obedecia ao seguinte cronograma: acelerôme- 
tros colocados às segundas, terças e quartas-feiras eram recolhidos na segunda-feira posterior à colocação do monitor; acelerômetros colocados às quintas, sextas e sábados eram recolhidos na quarta-feira posterior à colocação do monitor. A estratégia utilizada para retorno dos monitores foi a contratação de motociclistas, que se dirigiam às residências das crianças e dos jovens, conforme data e horário previamente combinados. Ao resgatar um conjunto de acelerômetros, os motociclistas entregavam os aparelhos para os responsáveis pelo download das informações e continuavam com o processo de busca, até completar as rotinas de trabalho às segundas $\mathrm{e}$ quartas-feiras. A equipe específica de acelerometria constituía-se de um auxiliar de pesquisa em tempo integral na sede, dois ou três motociclistas e alunos de doutorado.

Em razão de um número reduzido de acelerômetros disponíveis em algumas etapas do trabalho de campo e a quantidade de crianças / jovens atendidos diariamente, alguns realizaram os demais procedimentos da pesquisa e saíram sem a colocação dos acelerômetros. Esses casos foram considerados como pendências (Figura 1) e à medida que havia acelerômetros disponíveis, eles eram contatados pelos integrantes da equipe e, após, os motociclistas realizavam a colocação do aparelho no domicílio.

Em ambos os acompanhamentos os indivíduos que não residiam em Pelotas durante o trabalho de campo não foram considerados elegíveis para a colocação do acelerômetro, bem como aqueles que apresentavam limitações físicas importantes (por exemplo, cadeirantes) ou problemas mentais severos. Outros critérios de elegibilidade eram particulares à coorte de 1993. Por exemplo, os acelerômetros não foram colocados em gestantes e em jovens que desempenhavam atividades laborais em locais em que não era permitido o uso de relógios ou quaisquer tipos de pulseiras (como padarias e indústrias alimentícias).

Após o retorno dos acelerômetros à sede, a equipe responsável pela acelerometria realizava o registro de chegada dos monitores e iniciava os procedimentos de extração de dados. Inicialmente, os dispositivos eram carregados por aproximadamente duas horas. Depois eram colocados em uma base específica para downloads dos arquivos em um computador. Concluído o processo de downloads, os acelerômetros estavam disponíveis para iniciar um novo ciclo. Semanalmente, os dados contidos no computador eram transferidos para discos rígidos externos para manter cópia de segurança dos arquivos. $\mathrm{O}$ tamanho de cada arquivo variou de 300 a 600 $\mathrm{MB}$, necessitando uma grande capacidade de memória para armazenamento. Uma descrição resumida de todo o processo de coleta de dados é apresentada na Figura 1.

\section{Resultados em ambos os acompanhamentos}

Durante o trabalho de campo da coorte de 2004, apenas um acelerômetro foi perdido e recuperado ao término do campo. No acompanhamento da coorte de 1993, três foram perdidos. O modelo de monitor de punho foi muito bem recebido pelas crianças, que frequentemente o associavam a alguma personagem de televisão, desenho animado ou cinema. Embora o estudo fosse específico para as crianças dessa coorte, diversas vezes foram recebidas ligações de outras mães se disponibilizando para participar da pesquisa, algo que se acentuou quando as crianças utilizaram o monitor no período escolar. Algumas crianças não localizadas em acompanhamentos anteriores da coorte foram redescobertas em função dessa receptividade ao "reloginho", forma como o acelerômetro foi popularmente difundido nessa faixa etária. Com os jovens de 18 anos, embora a popularidade do monitor não tenha sido uma característica preponderante, sua aceitação foi considerada adequada à maioria dos participantes. Ainda que não tenha sido realizado um estudo de aceitabilidade, poucos foram os casos em que os jovens se negaram a colocar o monitor. Em algumas circunstâncias pontuais, solicitavam permissão para retirar o aparelho em determinados eventos como festas de formatura, aniversário e casamento.

A coorte de 2004 acompanhou um total de 3.721 crianças. Ao realizar o somatório de crianças acompanhadas ao número de mortes até o período (contabilidade adequada aos estudos de coorte), a coorte alcançou $90,2 \%$ de sua população elegível. Neste estudo a sede recebeu um total de 3.556 crianças, contudo, em alguns casos, as crianças foram visitadas em casa e essa parcela do acompanhamento não foi coberta pelo estudo de acelerometria. Assim, o denominador válido para a acelerometria foi o número de crianças que visitaram a clínica, e dessa forma, 89,5\% das crianças fizeram uso do acelerômetro (Tabela 1).

A coorte de 2004 coletou 514 dados com o modelo Genea, entretanto, devido aos problemas com a memória do aparelho, dificuldade com os downloads, pulseiras inadequadas e fragilidade dos monitores, houve substituição gradativa deles para o modelo GENEActiv, o qual foi utilizado até o fim do acompanhamento. Em termos gerais, o novo monitor compensou os problemas do anterior e foi mais resistente ao trabalho 
Descrição dos resultados dos acompanhamentos relacionados à acelerometria. Coortes de nascimentos de Pelotas, Rio Grande do Sul, Brasil, 1993 e 2004.

\begin{tabular}{lccccc}
\hline Coorte & $\begin{array}{c}\text { Faixa etária } \\
\text { (anos) }\end{array}$ & $\begin{array}{c}\text { Período de } \\
\text { trabalho de campo }\end{array}$ & $\begin{array}{c}\text { N total } \\
\text { da coorte }\end{array}$ & $\begin{array}{c}\text { N total do de } \\
\text { acompanhamento } \\
\text { (\%) }\end{array}$ \\
\hline 2004 & $6-7$ & 10 meses & 4.137 & $3.816(90,2)$ & 3.331 \\
1993 & 18 & 7 meses & 5.249 & $4.106(81,4)$ & 3.816 \\
\hline
\end{tabular}

* Indicador específico para cada acompanhamento conforme critérios de elegibilidade.

duradouro e repetitivo de uma coleta populacional. A empresa responsável pelos aparelhos disponibilizou o modelo GENEActiv durante o acompanhamento da coorte de 2004. Dessa forma, esta coorte utilizou os dois aparelhos.

No estudo da coorte de 1993, foram acompanhados 4.106 indivíduos, totalizando um percentual de acompanhamento de $81,4 \%$ dos nascidos vivos em Pelotas em 1993. Entre os acompanhados, 26 recusaram utilizar o monitor e 276 atendiam a critérios de exclusão. Portanto, 3.822 acelerômetros foram colocados, cobrindo um total de 99\% dos jovens elegíveis e 92,9\% dos acompanhados. Nesse acompanhamento foi utilizado apenas o monitor GENEActiv. Na coorte de 1993 todos os jovens que atendiam aos critérios de elegibilidade foram procurados para colocação dos acelerômetros (Tabela 1).

\section{Discussão}

Pesquisas epidemiológicas na área de saúde pública que envolvem atividade física utilizam majoritariamente questionários como ferramenta empírica. Com o intuito de obter medidas objetivas de atividade física, os acelerômetros são utilizados como uma possibilidade neste campo do conhecimento. Sabe-se que a maioria dos estudos envolvendo acelerometria não contempla grandes amostras populacionais, à exceção de estudos como o National Health and Nutritional Examination Survey (NHANES), com 6.329 crianças, jovens e adultos 13 e o Canadian $\mathrm{He}$ alth Measures Survey (CHMS) avaliando 4.440 indivíduos de 06 a 79 anos 14,15. Dentre os motivos que explicam a carência de estudos com grandes amostras, estão o relativo alto custo dos monitores e a logística complexa de colocação dos aparelhos, que envolve grande demanda de profissionais, além de conhecimentos técnicos específicos, seja com o modelo, ou software, ou análise dos dados.
No presente estudo, durante a realização dos acompanhamentos, utilizaram-se aproximadamente 200 monitores em uma rotina normal de funcionamento. Por causa das dificuldades logísticas relativas aos acelerômetros como quebras, perdas, falhas na recarga ou processamento de informações e pulseiras inadequadas, cerca de 400 acelerômetros foram necessários ao longo dos dois acompanhamentos. Assim, o suporte para a reposição dos equipamentos com defeito, possibilitado pelo convênio, foi fundamental à manutenção da coleta de dados de acelerometria. Além disso, ressaltam-se a necessidade de um armazenamento e backup adequado dos arquivos gerados pelos acelerômetros e a constituição de uma equipe com ampla dedicação para o desenvolvimento desse tipo de logística, que é complexa e varia constantemente, de acordo com os dias de coleta dos monitores e os diferentes períodos de um trabalho de campo. A comparabilidade do acelerômetro utilizado neste estudo com outras evidências da literatura passa a ser um desafio para futuras investigações. O recente estudo de calibração conduzido por Phillips et al. 16, em crianças de 8 a 14 anos, indicou pontos de corte para diferentes atividades físicas, o que reforça o crescimento e aceitação acadêmica dos tipos de acelerômetros aqui apresentados.

Ofertar aos pares acadêmicos estratégias para condução de estudos de alta complexidade logística tem sido uma conduta frequente na literatura. No Canadá, estudo com crianças de 03 a 05 anos descreve metodologia de atividade física e aptidão física a ser coletada em uma série de acompanhamentos ao longo de três anos. Nesse estudo, a acelerometria será uma das estratégias a serem empregadas 17 . Na mesma perspectiva, foram publicados os procedimentos metodológicos da coorte The Avon Longitudinal Study of Parents and Children (ALSPAC), que acompanha até os dias atuais os nascidos entre abril de 1991 e dezembro de 1992 da região de Avon, Inglaterra, descrevendo entre outros protocolos a men- 
suração de atividade física por meio de acelerometria 18. Publicações como essas podem ser incentivadoras de novas coletas e auxiliam no planejamento de estudos em outros contextos.

Uma reflexão necessária é a existência de uma "evolução tecnológica" no campo e uma espécie de corrida mercadológica para cobrir os interesses de pesquisa e produção de conhecimento. A busca intensa por dados mais acurados e precisos de atividade física fomenta uma lógica de concorrência em que as empresas oferecem modelos cada vez mais sofisticados, causando um impacto científico, visto que é evidente a expansão de trabalhos de validação e de técnicas dos novos modelos lançados no mercado. Yang \& Hsu 19 apresentam uma relação detalhada do funcionamento de sete modelos de acelerômetros (SenseWear, CT1/RT3, AMP331, GT3X/ GT1M, StepWatch, activPAL e IDEEA). Essa revisão descreve as características de tamanho, peso, local de uso, epoch, tipo e duração de bateria, capacidade de memória, entre outros. Ao mesmo tempo em que não há um modelo definitivamente superior aos outros, cabe colocar também que os acelerômetros " não estão livres de problemas", como enfatizou Janz 20 . Conforme o autor, as medidas objetivas devem se somar aos questionários e às técnicas analíticas de tratar os dados, pois com a corrida pelas formas objetivas de medição de atividade física não pode ser esquecida a complexidade para mensurar tal comportamento. As principais dificuldades apresentadas na acelerometria incluem a menor capacidade de captar movimentos do plano superior do corpo, incapacidade de diferenciar os domínios e contextos mensurados, problemas técnicos específicos como descalibragem, descarregamento, medidas de difícil interpretação e algumas impossibilidades de mensuração como no caso do carregamento de peso 20.

Há diversas preocupações relevantes sob a ótica das decisões metodológicas, dado que os diferentes modelos existentes implicam uma multiplicidade de escolhas e decisões em cada estudo. Exemplos como o número de dias a ser utilizado em situação de pesquisa varia conforme a faixa etária e não está definitivamente estabelecido na literatura. Por exemplo, com crianças, Trost 21 descreve que seria necessária a utilização de acelerometria por quatro a cinco dias para se ter uma medida confiável de atividade física. Por outro lado, em revisão mais recente 22 o mesmo autor preconiza um acompanhamento de quatro a nove dias nessa faixa etária. Nos acompanhamentos relatados no presente estudo, o período de uso foi de cinco a oito dias. A decisão se baseou na ideia de registrar a atividade física em pelo menos um dia da semana e de fim de semana (conforme esquema demonstrado na Figura 1). Com relação aos eixos captados, componente variável conforme modelo utilizado, mensurações em três eixos podem representar de forma mais acurada os padrões de atividade física em comparação a medidas uniaxiais.

$\mathrm{O}$ arcabouço metodológico aqui apresentado, ao serem estudados mais de 7.000 indivíduos com acelerômetro de punho, dentre eles crianças e adolescentes, tem o intuito de encorajar o planejamento de novos estudos populacionais no tema. Abre-se uma ferramenta com possibilidades e interesse em dialogar com outras experiências no campo da epidemiologia da atividade física, fomentando futuros estudos para comparação de dados de acelerometria em diferentes contextos. Foi descrita uma série de elementos indicando a complexidade e a dificuldade logística de estudos tão amplos, no entanto, a elevada adesão dos participantes foi uma surpresa positiva, já que tal experiência é inovadora e não se sabia como seria a aceitação dos indivíduos estudados. A coorte de nascidos em 1982 será a próxima a experimentar coleta desse porte, quando seus participantes estiverem com 30 anos (em 2012). Com a mudança na faixa-etária, certamente novos conhecimentos e implicações logísticas surgirão. 


\section{Resumen}

El objetivo de este estudio es caracterizar la metodología de recolección de la actividad física por acelerometría en las cohortes de nacidos en 2004 y 1993 en Pelotas, Río Grande do Sul, Brasil, en un seguimiento de 6-7 y 18 , respectivamente. Al visitar la sede de un centro de salud general, estos sujetos recibieron el acelerómetro y la posterior encuesta en el domicilio se llevó a cabo por los responsables del equipo de investigación. Los modelos utilizados fueron el Genea y GENEActiv, estimadores triaxiales de la aceleración de la gravedad (g), que se utilizaron en niños y adolescentes durante un período de 5 a 8 días. El número de individuos con datos de acelerometría en las cohortes fue de 3.331 niños (93,7\% de seguimiento) y 3.816 jóvenes $(92,9 \%$ de seguimiento). Con el fin de caracterizar la recolección de acelerometría en más de 7.000 individuos, se presenta un marco metodológico para el diseño de nuevos estudios de población sobre el tema, describiendo situaciones específicas de la experiencia y calificando la comprensión de la actividad física en el contexto de los estudios epidemiológicos.

Estudios de Cohortes; Actividad Motora; Metodología

\section{Colaboradores}

A. G. Knuth, V. V. Ramires, I. C. M. Silva e P. C. Hallal participaram da coleta de dados e da revisão e escrita final. M. C. F. Assunção, H. Gonçalves, A. M. B. Menezes, I. S. Santos, A. J. D. Barros e A. Matijasevich revisaram e aprovaram a versão final.

\section{Agradecimentos}

Agradecemos a Wellcome Trust, pelo financiamento ao projeto Implications of Early Life and Contemporary Exposures on Body Composition, Human Capital, Mental Health and Precursors of Complex Chronic Diseases in Three Brazilian Cohorts (1982, 1993 and 2004) (no 086974/Z/08/Z). Fases anteriores das coortes de nascimento de Pelotas foram financiadas pela Organização Mundial da Saúde (OMS), pelo Programa de Apoio a Núcleos de Excelência do Conselho Nacional de Desenvolvimento Científico e Tecnológico (PRONEX/CNPq).

\section{Referências}

1. Sirard JR, Pate RR. Physical activity assessment in children and adolescents. Sports Med 2001; 31:439-54.

2. Esliger DW, Rowlands AV, Hurst TL, Catt M, Murray P, Eston RG. Validation of the GENEA Accelerometer. Med Sci Sports Exerc; 43:1085-93.

3. Reichert FF, Menezes AM, Kingdom Wells JC, Ekelund E, Rodrigues FM, Hallal PC. A methodological model for collecting high-quality data on physical activity in developing settings-the experience of the 1993 Pelotas (Brazil) Birth Cohort study. J Phys Act Health 2009; 6:360-6.

4. Barros AJ, da Silva dos Santos I, Victora CG, Albernaz EP, Domingues MR, Timm IK, et al. The 2004 Pelotas birth cohort: methods and description. Rev Saúde Pública 2006; 40:402-13.

5. Barros AJ, Santos IS, Matijasevich A, Araujo CL, Gigante DP, Menezes AM, et al. Methods used in the 1982, 1993, and 2004 birth cohort studies from Pelotas, Rio Grande do Sul State, Brazil, and a description of the socioeconomic conditions of participants' families. Cad Saúde Pública 2008; 24 Suppl 3:S371-80.
6. Barros FC, Victora CG, Horta BL, Gigante DP. Methodology of the Pelotas birth cohort study from 1982 to 2004-5, Southern Brazil. Rev Saúde Pública 2008; 42 Suppl 2:7-15.

7. Santos IS, Barros AJ, Matijasevich A, Domingues MR, Barros FC, Victora CG. Cohort profile: the 2004 Pelotas (Brazil) birth cohort study. Int J Epidemiol 2011; 40:1461-8.

8. Victora CG, Hallal PC, Araujo CL, Menezes AM, Wells JC, Barros FC. Cohort profile: the 1993 Pelotas (Brazil) birth cohort study. Int J Epidemiol 2008; 37:704-9.

9. Hallal PC, Bertoldi AD, Goncalves H, Victora CG. Prevalência de sedentarismo e fatores associados em adolescentes de 10-12 anos de idade. Cad Saúde Pública 2006; 22:1277-87.

10. Domingues MR, Barros AJ, Matijasevich A. Leisure time physical activity during pregnancy and preterm birth in Brazil. Int J Gynaecol Obstet 2008; 103:9-15.

11. Dumith SC, Hallal PC, Menezes AMB, Araujo CL. Sedentary behavior in adolescents: the 11-year follow-up of the 1993 Pelotas (Brazil) birth cohort study. Cad Saúde Pública 2010; 26:1928-36. 
12. Hallal PC, Wells JC, Reichert FF, Anselmi L, Victora CG. Early determinants of physical activity in adolescence: prospective birth cohort study. BMJ 2006; 332:1002-7.

13. Troiano RP, Berrigan D, Dodd KW, Masse LC, Tilert T, McDowell M. Physical activity in the United States measured by accelerometer. Med Sci Sports Exerc 2008; 40:181-8.

14. Colley RC, Garriguet D, Janssen I, Craig CL, Clarke J, Tremblay MS. Physical activity of Canadian children and youth: accelerometer results from the 2007 to 2009 Canadian Health Measures Survey. Health Rep 2011; 22:15-23.

15. Colley RC, Garriguet D, Janssen I, Craig CL, Clarke J, Tremblay MS. Physical activity of Canadian adults: accelerometer results from the 2007 to 2009 Canadian Health Measures Survey. Health Rep 2011; 22:7-14.

16. Phillips LR, Parfitt G, Rowlands AV. Calibration of the GENEA accelerometer for assessment of physical activity intensity in children. J Sci Med Sport 2012; [Epub ahead of print].

17. Timmons BW, Proudfoot NA, MacDonald MJ, Bray SR, Cairney J. The health outcomes and physical activity in preschoolers (HOPP) study: rationale and design. BMC Public Health 2012; 12:284.
18. Fraser A, MacDonald-Wallis C, Tilling K, Boyd A, Golding J, Davey Smith G, et al. Cohort profile: the Avon Longitudinal Study of Parents and Children: ALSPAC mothers cohort. Int J Epidemiol 2012; [Epub ahead of print].

19. Yang C-C, Hsu Y-L. A review of accelerometrybased wearable motion detectors for physical activity monitoring. Sensors (Basel) 2010; 10:7772-88.

20. Janz KF. Physical activity in epidemiology: moving from questionnaire to objective measurement. Br J Sports Med 2006; 40:191-2.

21. Trost SG. Objective measurement of physical activity in youth: current issues, future directions. Exerc Sport Sci Rev 2001; 29:32-6.

22. Trost SG, McIver KL, Pate RR. Conducting accelerometer-based activity assessments in fieldbased research. Med Sci Sports Exerc. 2005; 37(11 Suppl):S531-43.

Recebido em 30/Mai/2012

Versão aprovada em 06/Out/2012

Aprovado em 31/Out/2012 